\title{
Pleistocene sea level fluctuation and host plant habitat requirement influenced the historical phylogeography of the invasive species Amphiareus obscuriceps (Hemiptera: Anthocoridae) in its native range
}

\author{
Danli Zhang ${ }^{1 \dagger}$, Zhen Ye ${ }^{1 *+}$, Kazutaka Yamada ${ }^{2 \dagger}$, Yahui Zhen ${ }^{1}$, Chenguang Zheng ${ }^{1}$ and Wenjun Bu ${ }^{1 *}$
}

\begin{abstract}
Background: On account of repeated exposure and submergence of the East China Sea (ECS) land bridge, sea level fluctuation played an important role in shaping the population structure of many temperate species across the ECS during the glacial period. The flower bug Amphiareus obscuriceps (Poppius, 1909) (Hemiptera: Anthocoridae) is an invasive species native to the Sino-Japanese Region (SJR) of East Asia. We tested the hypothesis of the ECS land bridge acting as a dispersal corridor or filter for A. obscuriceps during the glacial period. Specifically, we tested whether and the extent to which dispersal ability and host plant habitat requirement influenced the genetic structure of A. obscuriceps during the exposure of the ECS land bridge.

Results: Phylogenetic and network analyses indicated that A. obscuriceps is composed of two major lineages, i.e., China and Japan. Divergence time on both sides of the ECS was estimated to be approximately $1.07(0.79-1.32) \mathrm{Ma}$, which was about the same period that the sea level increased. No significant Isolation by Distance (IBD) relationship was found between $\Phi$ st and Euclidean distances in the Mantel tests, which is consistent with the hypothesis that this species has a good dispersal ability. Our Last Glacial Maximum (LGM) niche modeling of plants that constitute preferred habitats for $A$. obscuriceps exhibited a similar habitat gap on the exposed ECS continental shelf between China and Japan, but showed a continuous distribution across the Taiwan Strait.

Conclusion: Our results suggest that ecological properties (habitat requirement and dispersal ability), together with sea level fluctuation during the Pleistocene across the ECS, have shaped the genetic structure and demographic history of $A$. obscuriceps in its native area. The host plant habitat requirement could also be a key to the colonization of the A. obscuriceps species during the exposure of the ECS land bridge. Our findings will shed light on the potential role of habitat requirement in the process of biological invasion in future studies.

Keywords: Dispersal ability, East China Sea, Cological niche modeling, Genetic structure, Habitat requirement, Last Glacial Maximum, Land bridge

(Continued on next page)
\end{abstract}

\footnotetext{
* Correspondence: yezhen1987331@nankai.edu.cn; wenjunbu@nankai.edu.cn

${ }^{\dagger}$ Equal contributors

${ }^{1}$ Institute of Entomology, College of Life Sciences, Nankai University, 94

Weijin Road, Tianjin 300071, China

Full list of author information is available at the end of the article
} 
(Continued from previous page)

Abbreviations: AIC, Akaike information criterion; AMOVA, Analysis of molecular variance; AUC, The areas under the curve; BGI, Beijing Genomics Institute; BI, Bayesian inference; BP, Before present; COI, Cytochrome c oxidase subunit I; COII, Cytochrome c oxidase subunit II; CytB, Cytochrome b; EBSP, Extended Bayesian skyline plots; ECS, The East China Sea; ENM, Ecological niche modeling; ESS, Effective sample size; GBIF, Global Biodiversity Information Facility; IBDWS, Isolation by Distance Web Service; IM, Isolation-with-migration; ITS1, Internal transcribed spacer 1; LGM, Last Glacial Maximum; LIG, Last Interglacial; MCMC, Markov Chain Monte Carlo; ML, Maximum likelihood; ROC, Receiver operating characteristic; SJR, Sino-Japanese region.

\section{Background}

The eastern areas of Sino-Japanese region stretch along more than $1500 \mathrm{~km}$ in an east-west direction, ranging from East China to Japan and the Korean Peninsula to Taiwan and the Ryukyu Islands [1]. Historically, this region has been heavily influenced by glacial-interglacial cycles during the Pleistocene, showing variations of coastal habitat availability and geomorphological patterns along the rim of the northwest Pacific [2]. During the LGM (approximately 22,000 years ago) of the last glacial period, wide extensions of continental shelf across the ECS were assumed to have been exposed, forming a large land bridge connecting the isolated continents of Mainland China, Japan, Taiwan, and probably the Korean Peninsula, because the sea levels were 85-140 $\mathrm{m}$ below the present levels [3]. Fossil pollen analyses and paleobiome reconstructions also indicate that several temperate plant species expanded across large areas of the continental shelf that emerged in the ECS as a consequence of sea level decline during the cold periods [4]. Phylogeographic structure and Pleistocene history of temperate species inhabiting the SJR were closely linked to the incidents of historical climatic changes associated with sea level fluctuations and land bridge configuration. The ECS land bridge, along with the sea level decline during the LGM, might have connected species or population distribution, either serving as a dispersal corridor, allowing continuous migration of many temperate species from the mainland China into Japan (or vice versa), or generating secondary contact or gene flow among the formerly isolated populations. This "dispersal corridor" hypothesis is supported by the extant distribution of several species, including the deciduous orient oak Quercus variabilis Blume, 1850 [5], the marine gastropod Thais clavigera (Küster, 1860) [6], and the semiaquatic insect Microvelia douglasi douglasi Scott, 1874 [7]. In these examples, a nearly homogenized genetic pattern was observed in the chloroplast/mitochondria/nuclear DNA sequences on both sides of the ECS land bridge (i.e., Mainland China and Japan). These observations are consistent with the large expanses of suitable spaces across the ECS land bridge reconstructed by paleoclimate niche modeling $[5,7]$.
In contrast, many studies provided evidence that the ECS land bridge also served as a dispersal filter that obstructed or even inhibited certain species or populations from migrating through the ECS land bridge during the LGM, as seen for two deciduous trees Platycrater arguta Siebold \& Zucc., 1835 [8] and Acer mono Maxim, 1856 [9]. This phenomenon suggests a high level of lineage differentiation on both sides of the ECS land bridge. A general interpretation of this genetic partition is that environment of the exposed ECS basin area was unsuitable for the survival of these species based on the evidence of LGM paleoclimate niche modeling reconstruction, which indicated that the ECS land bridge probably imposed a formidable barrier to dispersal during the glacial period [8]. However, this divergence interpretation emphasizes the external environment (i.e., glacial condition) but ignores the ecological properties of the species, including their dispersal abilities, habitat requirements, life histories and other factors. In particular, dispersal ability and habitat requirement are expected to have a strong influence on shaping the current population genetic structure [10]. Recent studies showed that, even if the suitable habitat was fragmented or discontinuous in some species distribution ranges, such geographical limitations could be overcome by species with moderate/strong dispersal abilities [11]. Many small animal species were also assumed to depend on their microhabitats, which could elude predators and supply adequate food and breeding places. This habitat requirement might restrict the species dispersal, shape a specific genetic structure, and even potentially derive new taxon from different lineages. For instance, studies on the reefassociated surgeon-fish, genus Acanthurus and the rock-associated lizard, genus Dalmatolacerta, indicate that habitat requirement is the main factor that shaped different phylogeographic patterns in these habitatassociated species $[12,13]$.

Few studies are focused on temperate invertebrate species of comprehensive samples, and the influence of dispersal ability and habitat requirement on the population genetic structure across the ECS are poorly understood. In this study, we focus on the flower bug Amphiareus obscuriceps (Poppius, 1909) (Hemiptera: Anthocoridae), 
a native species across the ECS land bridge $[14,15]$. This species can be found in various plant microhabitats where they prey on small anthropods, principally inhabiting in the dead leaf clusters of deciduous trees and shrub [14]. In its native range, most $A$. obscuriceps were usually collected from the deciduous trees, Castanea mollissima Blume, 1851, Salix babylonica L., 1753, Salix matsudana Koidz, 1915, and the shrub, Sorbaria sorbifolia (L.) A. Braun, 1860 [14], all of which are native to East Asia [16]. Recently, A. obscuriceps has become an invasive species that is rapidly spreading in North America and Europe [15, 17, 18]. Considering its host plant habitat requirement and potentially moderate/strong dispersal ability like other invasive species, A. obscuriceps provides an ideal model for examining the phylogeographic structure and Pleistocene history associated with sea level fluctuation and ecological property across the ECS during the glacial period. We used a comprehensive sampling of $A$. obscuriceps in its native area, and applied ecological niche modeling (ENM) and multilocus genetic markers to infer a more robust phylogeographic conclusion of this species across the ECS. Specifically, this study aimed to (1) describe the phylogeographic structure; (2) infer the divergence time and historical demography; (3) test the hypothesis of ECS land bridge as a dispersal corridor or filter for A. obscuriceps in its native area during the last glacial period; and (4) evaluate the role of habitat requirement and dispersal ability in driving the current genetic structure during the exposure of the ECS land bridge in LGM period.

\section{Methods}

Sampling and laboratory procedures

We sampled 23 populations, comprising a total of 188 individuals in its native areas covering Mainland China, Taiwan Island and Japan (Fig. 1). Amphiareus ruficollaris Yamada \& Hirowatari, 2003 was used as the outgroup for our phylogenetic analysis. All samples were preserved in $95 \%$ ethanol and stored in a freezer at $-20{ }^{\circ} \mathrm{C}$ in the College of Life Sciences at Nankai University (Tianjin, China). Genomic DNA was extracted from the entire body, excluding the abdomen and genitalia using a General AllGen Kit (Qiagen, Germany). All individuals were analyzed by sequencing for the mitochondrial markers, cytochrome c oxidase subunit I (COI), cytochrome c oxidase subunit II (COII), and cytochrome b $(\mathrm{CytB})$ and the nuclear marker internal transcribed spacer 1 (ITS1). PCR primers designed in the present study are listed in Table S1 (Additional file 1: Table S1). The PCR program included an initial denaturation at $94{ }^{\circ} \mathrm{C}$ for $2 \mathrm{~min}$, followed by $31-33$ cycles of $30 \mathrm{~s}$ at $92^{\circ} \mathrm{C}, 30 \mathrm{~s}$ at $50-52{ }^{\circ} \mathrm{C}$, and $1 \mathrm{~min}$ at $72{ }^{\circ} \mathrm{C}$, ending with a final extension at $72{ }^{\circ} \mathrm{C}$ for $8 \mathrm{~min}$. PCR products were sent to BGI (Beijing Genomics Institute) where sequencing was conducted in both directions using a HiSeq 2000 sequencing system. Sequences were aligned with ClustalW [19] multiple alignment under default settings and visually proofread in Bioedit v7.0 software [20].

\section{Phylogenetic analysis}

Gene tree reconstructions of concatenated mitochondrial haplotypes were performed using Bayesian inference (BI)

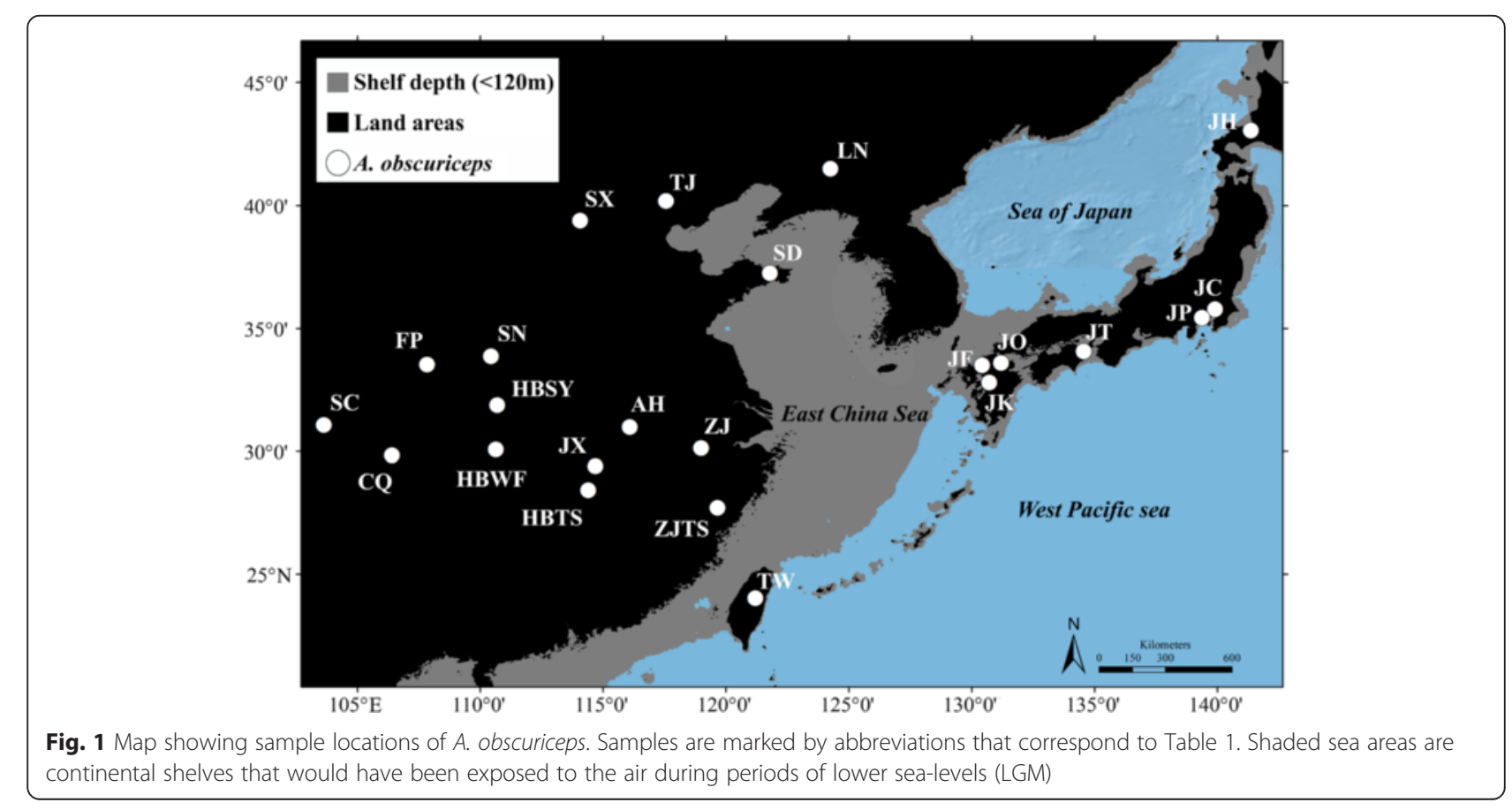


and Maximum likelihood (ML) methods, and implemented in MrBayes 3.2.5 [21] and raxmlGUI 1.5 [22] respectively. The program PartitionFinder 1.1.1 [23] was used to infer the best partitioning strategy and models of substitution for each gene in Bayesian and ML analyses. We used the Akaike information criterion (AIC) and the "greedy" algorithm with branch lengths estimated as "unlinked" to search for the best-fit scheme. For the Bayesian analysis, MrBayes 3.2.5 [21] was employed to reconstruct the phylogenetic trees under the HKY substitution model with a gamma distribution (+ G) and a proportion of invariable sites $(+\mathrm{I})$ to account for among-site rate variation. Two simultaneous runs of $10,000,000$ generations were conducted for concatenated matrix. Trees inferred prior to stationarity (at $25 \%$ of the run) were discarded as burn-in, and the remaining trees were used to construct a $50 \%$ majority-rule consensus tree. ML analysis was conducted with raxmlGUI 1.5 [22] under the substitution model GTRGAMMAI and the node support values were assessed by bootstrap resampling calculated using 1000 replicates.

\section{Genetic diversity and phylogeographic analysis}

Genetic diversities of mitochondrial and nuclear data were estimated for all populations and for each population using the number of polymorphic sites $(S)$, number of haplotypes (Nhap), haplotype diversity $(H d)$, and nucleotide diversity $(\pi)$, which were all calculated in DNASP 4.0 [24]. The algorithm of haplotype generation considered the sites with gaps/missing data. Network 4.6.1.3 (Fluxus Technology, Suffolk, UK) was used to create intraspecific median-joining networks to visualize the evolutionary relationships between haplotypes [25]. We also assessed the hierarchical partitioning of genetic structure between specimens from China and those from Japan, based on K2P distance by analyzing molecular variance (AMOVA) in Arlequin 3.5 [26]. Only the populations with more than 5 individuals were analyzed. The Mantel test was conducted in Isolation by Distance Web Service (IBDWS) using 1000 randomizations to test the isolation-bydistance model [27]. Finally, the potential geographical zones associated with genetic discontinuities across the entire sampling regions were further investigated by using Monmonier's algorithm implemented in Barrier 2.2 [28].

\section{Coalescent inferences of demographic history}

Demographic history was initially examined using two different approaches. First, mismatch distribution was used to identify recent demographic expansion and population equilibrium processes [29]. Mismatch distribution defined groups of populations by haplotype networks, and the statistical charts were calculated based on a population growth-decline model in DNASP 4.0 [24]. Then, three neutrality tests, Tajima's $D$, Fu and Li's $D^{*}$, and Fu's FS, which were implemented in DNASP 4.0 [24] or ARLEQUIN 3.5 [26], were performed. Extended Bayesian skyline plots (EBSP) were implemented in BEAST 1.8.2 [30] to estimate the shape of population growth through time with coupled multilocus data, which reduced estimation errors associated with single gene and increased the power of detecting demographic dynamics [31]. Analyses were conducted across the multilocus datasets (COI, CytB, and ITS1) by using a relaxed uncorrelated lognormal molecular clock. Given that the mutation rates for this species are unknown, we used the following confidence intervals from Reduviidae (Hemiptera: Cimicomorpha): COI, 0.6-1.0 \%/ Ma [32, 33]; CytB, 1.1-1.8 \%/Ma [34]; and ITS1, 0.4$1.0 \% / \mathrm{Ma}$ [35]. The data set was partitioned by genes and Modeltest was used to determine the best-fit model under the AIC for each gene [36]. We ran the chains for $100,000,000$ generations, and gene trees were saved every 1000 generations. EBSP coalescent model using linear type and the ploidy type had been properly setting. Convergence of the Markov Chain Monte Carlo (MCMC) chains was inspected using Tracer 1.6 [37] by visually checking that the effective sample size (ESS) for all relevant parameters were well above 200 .

\section{Estimation of divergence time and migration rate}

We estimated the divergence time $(t)$ and migration rate (m) using the isolation-with-migration (IM) model implemented in IMa2 [38]. In contrast to BEAST, the Bayesian approach in IMa2 generated a more realistic model because it considered possible gene flows among populations after they split from an ancestral species [39]. We used mitochondrial sequences for the IM analysis. The three component groups (i.e., Mainland China, Japan, and Taiwan) were divided according to the results of their haplotype networks. We initially conducted multiple short runs with an increasing number of steps, wide priors, and heating schemes to ensure that the complete posterior distribution could be obtained. The HKY model with 0.25 as the inheritance scalar was used, and the upper prior bounds were set to 20 for the population size $(q), 10$ for the divergence time $(t)$, and 1 for the migration rate $(m)$. Parameters were estimated based on mutation rates for COI $\left(0.6 \times 10^{-8}-1.0 \times 10^{-8}\right)$ and CytB $\left(1.1 \times 10^{-8}-1.8 \times 10^{-8}\right)$ per site per generation [32-34], and average generation time was set to 0.5 years according to previous studies of other Anthocoridae species [40]. Final runs consisted of $1 \times 100,000,000$ steps with a burn-in of $1 \times 10,000,000$ steps, and the lowest ESS among the parameters was greater than 50. In addition, we also used BEAST 1.8.2 [30] to estimate divergence time. The data set was partitioned by genes and was set specific clock per gene (COI and CytB). We used a coalescent exponential growth model with the chains run for $100,000,000$ generations, which was 
checked to make sure the ESS was more than 200 and the first $10 \%$ discarded as burn-in.

\section{Paleoclimate niche modeling reconstruction}

A total of 52 occurrence records of A. obscuriceps in its native area were used for niche modeling, including 23 records from the alcohol-preserved specimens and 29 records from pinned specimens deposited in Nankai University or the published literature. Model geographic background was a squared area $(\mathrm{Y} \max =45, \mathrm{Y} \min =17$, $\mathrm{X} \max =145, \mathrm{X} \min =100$ ) delimited by a bounding box containing all known native range occurrences of $A$. obscuriceps typically used in former studies [41]. Meanwhile, we also used host plants C. mollissima, Sa. babylonica, Sa. matsudana and So. sorbifolia [14] for niche modeling to examine the degree to which the distribution of the related host plant species of $A$. obscuriceps have changed since the Pleistocene glaciation. The host plant coordinates in its native area for niche model building were downloaded from the Global Biodiversity Information Facility online database (GBIF, http://www.gbif.org/), which determined 335 localities for C. mollissima, 93 for Sa. babylonica, 59 for Sa. matsudana, and 44 for So. sorbifolia. We chose bioclimatic variables to represent annual trends and extreme or limiting conditions, because many taxa are limited by environmental extremes [41]. Seven bioclimatic variables, which were most likely to restrict the distribution of A. obscuriceps and related host plants, were selected from the WorldClim database [42] (http://www.worldclim.org/), including mean temperature (BIO1), mean diurnal temperature range (BIO2), maximum temperature of the warmest month (BIO5), minimum temperature of the coldest month (BIO6), annual mean precipitation (BIO12), precipitation of the wettest month (BIO13), and precipitation of the driest month (BIO14). We conducted an autocorrelation test of the 7 bioclimate variables that showed relatively low Spearman's coefficients $(r<0.80)$ (Additional file 2: Table S2). All the variables were derived from the WorldClim data centre at a resolution of 2.5-arc. We used the maximum entropy implemented in Maxent 3.3.3 k [43] to develop the current distribution model at 2.5 arc-min resolution. The analysis was run using the default program conditions, with $75 \%$ of the recorded species for training and $25 \%$ for testing the model. The model was then projected onto the set of climatic variables simulated by the Community Climate System Model 3 [44] to infer the extent of suitable areas during the LGM. The areas under the curve (AUC) of the "receiver operating characteristic (ROC)" plot were used for model evaluation [45]. The same seven environmental variables were used to assess global areas of potential invasion. We used 52 occurrences of the native localities for model construction and applied their prediction onto the invasive continents. We compared areas of potential invasion with the actual records in Europe and North America for model evaluation. Discoveries of $A$. obscuriceps in different countries were summarized, and 135 actual invasion records in the US and Europe were obtained from published studies (Additional file 3: Table S3). In addition, we also collected all the coordinates of the four host plants from the GBIF outside the native range, and positioned the actual records to the map of the predicted A. obscuriceps invasive areas.

\section{Results}

\section{Genetic diversity and phylogenetic analysis}

For mitochondrial DNA, 1584 bp of protein-coding regions were obtained from 188 individuals, including segments of the COI (633 bp), COII (540 bp) and CytB (411 bp) genes. A total of 182 unique haplotypes were derived from tandem sequence $(\mathrm{COI}+\mathrm{COII}+\mathrm{CytB})$ among all individuals. The 277 polymorphic sites included 107 singleton variables and 170 parsimony informative sites. The nucleotide and haplotype diversities ranged from 0.00168 to 0.01199 (mean 0.01926) and from 0.667 to 1.000 (mean 0.9995 ) respectively (Table 1 ). Both BI and ML phylogenetic analyses of $A$. obscuriceps included two major lineages (i.e., China and Japan) (Additional file 4: Figure S1 and Additional file 5: Figure S2). Individuals from Taiwan were structurally nested within the Chinese lineage (Additional file 4: Figure S1 and Additional file 5: Figure S2).

\section{Phylogeographic analysis}

When the 182 haplotypes were used for network analysis, two independent haplogroups (i.e., China and Japan) with higher levels of reticulation were identified. A similar topological structure was recognized using the single COII dataset to reduce the complexity of reticulation and achieve clear exhibition (Fig. 2a). The overall network had two independent star-like haplogroups (China and Japan) and several individuals from Taiwan showed nested structures within the haplogroup of China (Fig. 2a), which indicated a typical population expansion [46]. AMOVA revealed a significant genetic variance when populations were grouped by phylogenetic lineages (i.e., China and Japan, $80.13 \%, p<0.01$ ), where approximately $18.75 \%$ of the genetic variation was within populations and only $1.12 \%$ was among populations within the group (Table 2). Mantel tests showed no significant IBD relationship between $\Phi s t$ and Euclidean distances in the two divergent lineages (Fig. 3), indicating that this species was not dispersal-limited and thus might possess a strong capacity for long-distance dispersal. Zones of genetic discontinuities identified by the Barrier 2.2 program showed a potential association of geographic barriers with genetic abruption (Additional file 6: Figure S3). 
Table 1 Nucleotide polymorphisms in each geographic population

\begin{tabular}{|c|c|c|c|c|c|c|c|}
\hline $\mathrm{COI}+\mathrm{COII}+\mathrm{CytB}$ & Lat. & Long. & Sample size & S & Nhap & $H d$ & $\pi$ \\
\hline \multicolumn{8}{|l|}{ Mainland China } \\
\hline $\mathrm{AH}$ & $30^{\circ} 58^{\prime} 57^{\prime \prime}$ & $116^{\circ} 4^{\prime} 49^{\prime \prime}$ & 9 & 46 & 9 & 1.000 & 0.00838 \\
\hline CQ & $29^{\circ} 50^{\prime} 16^{\prime \prime}$ & $106^{\circ} 23^{\prime} 48^{\prime \prime}$ & 10 & 47 & 10 & 1.000 & 0.00770 \\
\hline FP & $33^{\circ} 31^{\prime} 51^{\prime \prime}$ & $107^{\circ} 49^{\prime} 45^{\prime \prime}$ & 10 & 40 & 10 & 1.000 & 0.00650 \\
\hline HBSY & $31^{\circ} 52^{\prime} 49^{\prime \prime}$ & $110^{\circ} 41^{\prime} 13^{\prime \prime}$ & 2 & 19 & 2 & 1.000 & 0.01199 \\
\hline HBTS & $29^{\circ} 23^{\prime} 57^{\prime \prime}$ & $114^{\circ} 40^{\prime} 51^{\prime \prime}$ & 1 & - & 1 & - & - \\
\hline HBWF & $30^{\circ} 4^{\prime} 40^{\prime \prime}$ & $110^{\circ} 37^{\prime} 33^{\prime \prime}$ & 1 & - & 1 & - & - \\
\hline$J X$ & $28^{\circ} 25^{\prime} 9^{\prime \prime}$ & $114^{\circ} 23^{\prime} 3^{\prime \prime}$ & 9 & 41 & 9 & 1.000 & 0.00733 \\
\hline LN & $41^{\circ} 29^{\prime} 51^{\prime \prime}$ & $124^{\circ} 14^{\prime} 56^{\prime \prime}$ & 12 & 45 & 12 & 1.000 & 0.00697 \\
\hline SC & $31^{\circ} 4^{\prime} 5^{\prime \prime}$ & $103^{\circ} 37^{\prime} 49^{\prime \prime}$ & 10 & 39 & 10 & 1.000 & 0.00647 \\
\hline SD & $37^{\circ} 14^{\prime} 34^{\prime \prime}$ & $121^{\circ} 46^{\prime} 41^{\prime \prime}$ & 9 & 22 & 9 & 1.000 & 0.00454 \\
\hline SN & $33^{\circ} 52^{\prime} 29^{\prime \prime}$ & $110^{\circ} 25^{\prime} 54^{\prime \prime}$ & 10 & 43 & 10 & 1.000 & 0.00735 \\
\hline$S X$ & $39^{\circ} 23^{\prime} 2^{\prime \prime}$ & $114^{\circ} 3^{\prime} 40^{\prime \prime}$ & 12 & 46 & 12 & 1.000 & 0.00962 \\
\hline TJ & $40^{\circ} 11^{\prime} 16^{\prime \prime}$ & $117^{\circ} 33^{\prime} 22^{\prime \prime}$ & 4 & 10 & 4 & 1.000 & 0.00358 \\
\hline ZJ & $30^{\circ} 7^{\prime} 54^{\prime \prime}$ & $118^{\circ} 59^{\prime} 4^{\prime \prime}$ & 14 & 32 & 13 & 0.989 & 0.00572 \\
\hline ZJTS & $27^{\circ} 42^{\prime} 29^{\prime \prime}$ & $119^{\circ} 39^{\prime} 4^{\prime \prime}$ & 8 & 32 & 8 & 1.000 & 0.00780 \\
\hline \multicolumn{8}{|l|}{ Japan } \\
\hline JC & $35^{\circ} 47^{\prime} 15^{\prime \prime}$ & $139^{\circ} 54^{\prime} 11^{\prime \prime}$ & 9 & 49 & 9 & 1.000 & 0.00775 \\
\hline$J F$ & $33^{\circ} 29^{\prime} 58^{\prime \prime}$ & $130^{\circ} 25^{\prime} 19^{\prime \prime}$ & 10 & 35 & 10 & 1.000 & 0.00540 \\
\hline$J \mathrm{H}$ & $43^{\circ} 3^{\prime} 44^{\prime \prime}$ & $141^{\circ} 21^{\prime} 16^{\prime \prime}$ & 6 & 29 & 6 & 1.000 & 0.00648 \\
\hline JK & $32^{\circ} 48^{\prime} 11^{\prime \prime}$ & $130^{\circ} 42^{\prime} 28^{\prime \prime}$ & 9 & 37 & 9 & 1.000 & 0.00614 \\
\hline JO & $33^{\circ} 35^{\prime} 54^{\prime \prime}$ & $131^{\circ} 11^{\prime} 18^{\prime \prime}$ & 10 & 32 & 10 & 1.000 & 0.00452 \\
\hline$J P$ & $35^{\circ} 26^{\prime} 35^{\prime \prime}$ & $139^{\circ} 21^{\prime} 45^{\prime \prime}$ & 3 & 4 & 2 & 0.667 & 0.00168 \\
\hline $\mathrm{JT}$ & $34^{\circ} 4^{\prime} 13^{\prime \prime}$ & $134^{\circ} 33^{\prime} 17^{\prime \prime}$ & 10 & 36 & 10 & 1.000 & 0.00574 \\
\hline \multicolumn{8}{|l|}{ Taiwan } \\
\hline TW & $24^{\circ} 1^{\prime} 22^{\prime \prime}$ & $121^{\circ} 11^{\prime} 14^{\prime \prime}$ & 10 & 51 & 10 & 1.000 & 0.01072 \\
\hline
\end{tabular}

$S$ number of segregating sites, $N$ Hap number of haplotypes, $H d$ haplotype diversity, $\pi$ nucleotide diversity

When $K=1$, the chief bold line first divided the entire sampling region into the China and Japan subregions, which reflected significant genetic/geographical isolation between them (Additional file 6: Figure S3a). When $K=2$, the second bold line separated Taiwan from Mainland China, indicating that the population from Taiwan also had a certain degree of differentiation from Mainland China populations (Additional file 6: Figure S3b).

For the nuclear sequence data, $418 \mathrm{bp}$ partial fragments of the ITS1 gene were successfully obtained from 178 individuals. A total of 10 unique haplotypes were derived from all individuals. The 10 polymorphic sites included 6 singleton variables and 4 parsimony informative sites. The nucleotide diversities and haplotype diversities ranged from 0 to 0.00292 (mean 0.00304) and from 0 to 0.711 (mean 0.664) respectively (Additional file 7: Table S4). Two haplogroups were also recognized in the MJ haplotype network (Fig. 2b), reflecting a significant genetic isolation between populations in China and Japan. The population in Taiwan included two haplotypes: one related to the ancestral haplotype of Mainland China, and the other a derivative haplotype (Fig. 2b). The star-like shapes in the network also indicated that $A$. obscuriceps experienced population expansion events (Fig. 2b). AMOVA indicated that the genetic variation was $83.50 \%$ among groups $(p<0.01)$, $0.52 \%$ among populations within groups, and $15.98 \%$ within populations (Table 2). The large differentiation indicated that allele frequencies were different between the samples from China and Japan. Barrier analysis also showed that major differentiation occurred between the populations from China and Japan $(K=1)$ (Additional file 6: Figure S3c), and the population from Taiwan showed slight divergence from Mainland China $(K=4)$ (Additional file 6: Figure S3d). 


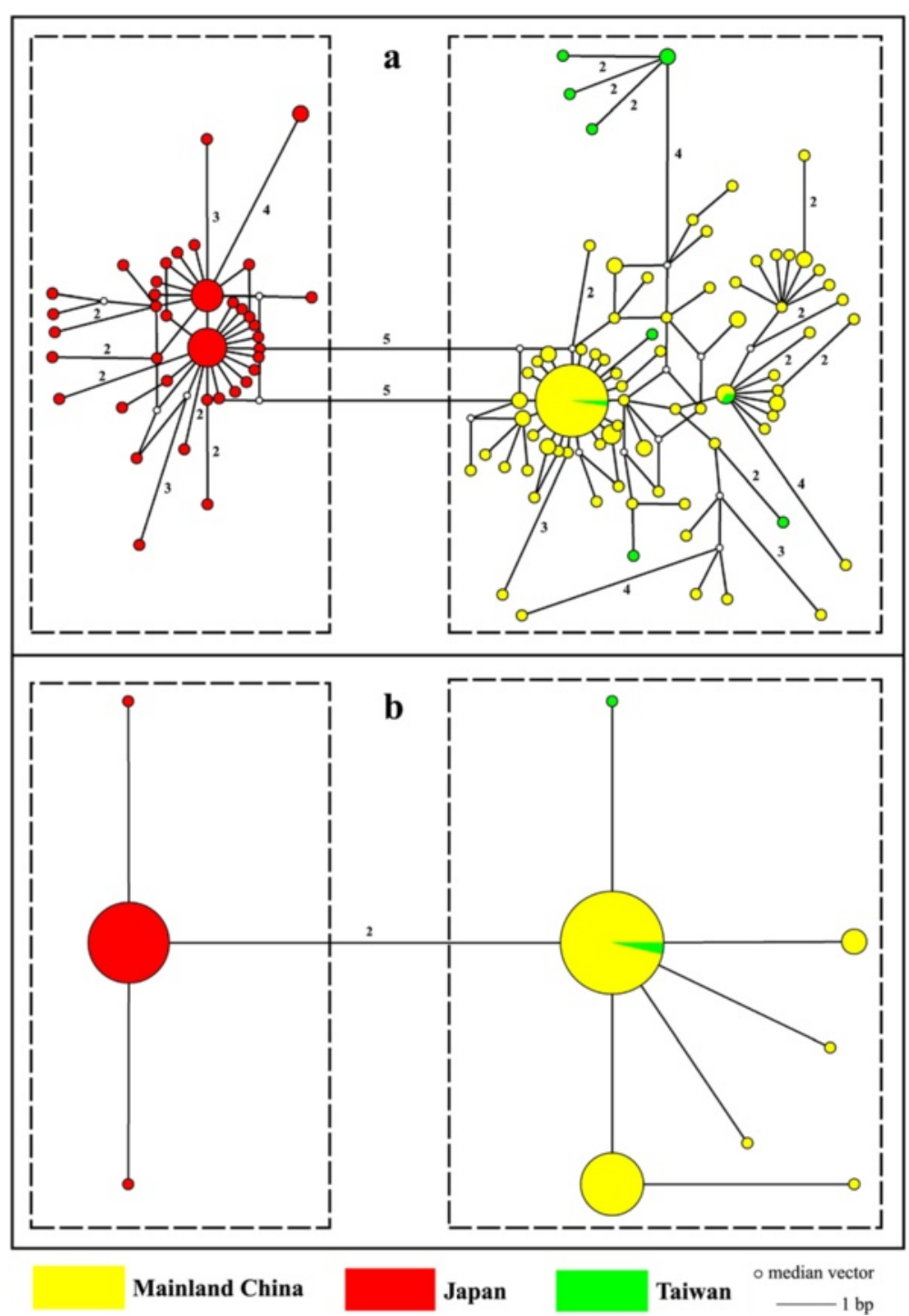

Fig. 2 Median joining haplotype network constructed using Network. Haplotype circle size denotes the number of sampled individuals. Colors correspond to different regions. Numbers of base pair changes (no number $=1$ bp) are given. a Mitochondrial data. b Nuclear data

Table 2 Hierarchical analyses of molecular variance for A. obscuriceps

\begin{tabular}{lllrrr}
\hline Genetic marker & Regional subdivisions & Source of variation & Variance explained & $P$ & Fixation index \\
\hline mtDNA & China \& Japan groups & Among group & 80.13 & 0.00 & 0.801 \\
& & Among populations within groups & 1.12 & 0.00 & 0.056 \\
& & Within populations & 18.75 & 0.00 & 0.812 \\
nrDNA & China \& Japan groups & Among group & 83.50 & 0.00 & 0.835 \\
& & Among populations within groups & 0.52 & 0.17 & 0.032 \\
& & Within populations & 15.98 & 0.00 & 0.840 \\
\hline
\end{tabular}



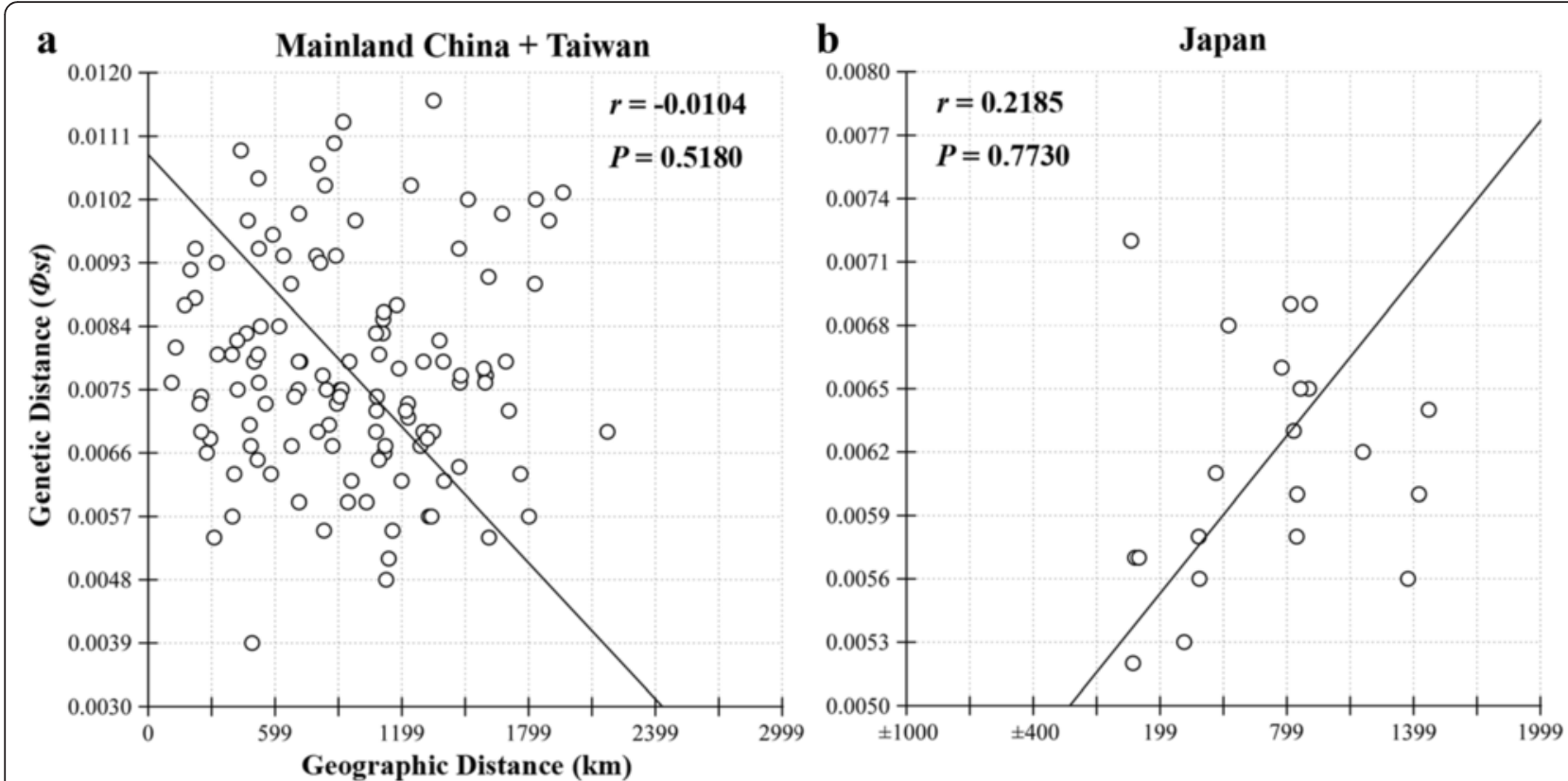

Fig. 3 Scatter plot showing the relationship between genetic distances ( $\Phi$ st) and geographical distances (km). a Lineages of Mainland China and Taiwan. b Lineage of Japan

\section{Coalescent inferences of demographic history}

Multimodal mismatch distributions are assumed to characterize old populations of constant size, whereas expanding populations are considered to be unimodal [47]. Mismatch distribution analyses revealed a multimodal distribution for the population from Taiwan (Fig. 4c), whereas a sudden expansion model with small and insignificant Harpending's raggedness index was obtained in Mainland China, Japan, and the entire sample regions $(r=0.00134-0.00565)$ (Fig. 4a, b \& d). The significantly negative values of Tajima's $D$, Fu's FS, and $\mathrm{Fu}$ and Li's $D^{*}$ (Table 3 ) indicated a scenario of demographic expansion in Mainland China, Japan, and all sample populations. Combined results of neutrality tests, the nonsignificant parameters and the multimodal mismatch distributions indicated a model of relatively constant population size in the population from Taiwan. The result of EBSP revealed a relatively explicit demographic history for the three regions (i.e., Mainland China, Japan, and Taiwan) and the entire set (Additional file 8: Figure S4). The results showed that the population from Taiwan kept a nearly stable and small population size (Additional file 8: Figure S4c), corresponding to its narrow and limited distribution, whereas the populations in Mainland China, Japan and the entire region showed a constantly increasing population size over time after a period of low-efficiency population sizes (Additional file 8: Figure S4a, b, d). After transforming the units of the $x$-axes to years, the population expansion of $A$. obscuriceps appeared to begin approximately $0.14 \mathrm{Ma}$ before present (BP).

\section{Divergence time and migration rate}

Simulation with IMa2 revealed unambiguous marginal posterior probability distributions of the demographic parameters. Divergence time was estimated to be 1.07 (95\% CI $=0.79-1.32$ ) Ma between the population in China and that in Japan and 0.15 (95 \% CI = 0.14-0.24) Ma between that in Mainland China and that in Taiwan. We also detected one statistically significant migration event of $A$. obscuriceps populations from Mainland China to Taiwan with $m=0.9995$, which was greater than zero and thus indicated that gene flow occurred during or soon after the divergence event. Estimation of the divergence time by BEAST was presented in Figure S5 (Additional file 9: Figure S5).

\section{Paleoclimate niche modeling}

The AUC of the ROC had values higher than 0.90 in all analyses, indicating a reasonable predictive model performance. Jackknife of regularized training gain for $A$. obscuriceps was shown in Figure S6 (Additional file 10: Figure S6). For A. obscuriceps, highly suitable areas were observed in the native range, which included most of East China, the Korean Peninsula and Japan (Fig. 5a). Integrating the current niche model into the simulated LGM climate conditions suggested that the potential range of the species contracted moderately toward the 


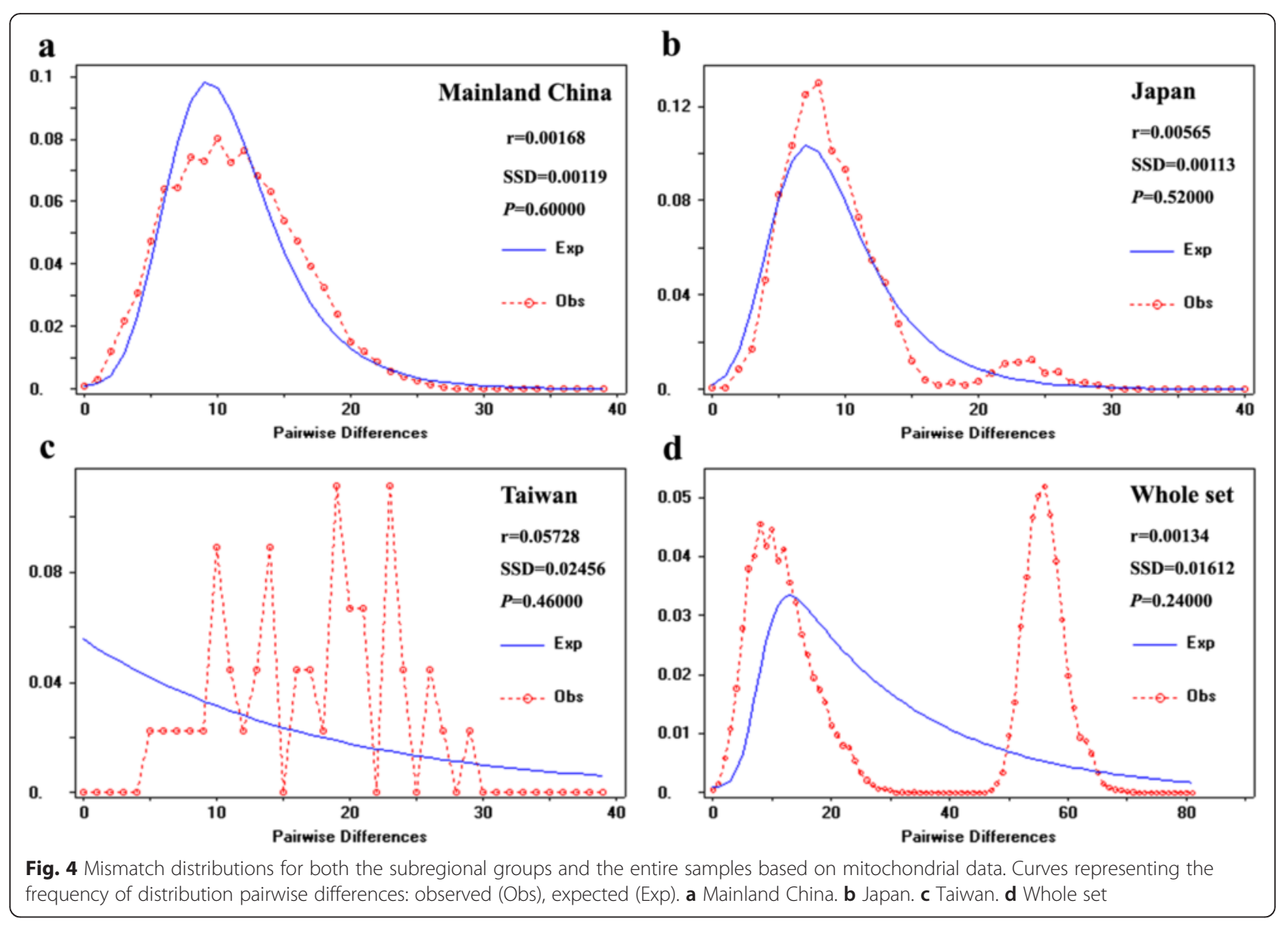

south. The highly suitable areas were mainly located in two segregated regions (Fig. 5b): the large-scale region distributed from southern China and extending to the continental shelf of the ECS across Taiwan and the narrow region located at the south coastal zone of Japan. The current potential areas of the four host plants of $A$. obscuriceps were also modeled according to their native areas. After projecting the current niche into the LGM historical climate condition, three of the 4 host plant

Table 3 Nucleotide polymorphisms and Neutrality tests in defined groups and whole dataset based on mitochondrial DNA data

\begin{tabular}{lllll}
\hline Parameter & Mainland China & Japan & Taiwan & Whole set \\
\hline Sample size & 121 & 57 & 10 & 188 \\
S & 179 & 135 & 51 & 277 \\
Nhap & 116 & 56 & 10 & 182 \\
Hd & 0.9989 & 0.9990 & 1.0000 & 0.9995 \\
$\pi$ & 0.00707 & 0.00598 & 0.01072 & 0.01926 \\
Tajima's D & $-2.24523^{* *}$ & $-2.42838^{* *}$ & -0.28481 & -1.30466 \\
Fu's Fs & $-24.27842^{* * *}$ & $-24.66929^{* * *}$ & -2.15008 & $-23.71411^{* *}$ \\
Fu and Li's D* & $-3.94409^{* *}$ & $-3.45690^{* *}$ & -0.56710 & $-3.94421^{* *}$
\end{tabular}

$S$, number of segregating sites; NHap, number of haplotypes; $H d$, haplotype diversity; $\pi$, nucleotide diversity. ${ }^{*} P<0.05 ;{ }^{* *} P<0.02 ;{ }^{* *} P<0.001$ species, C. mollissima, Sa. babylonica and Sa. matsudana, exhibited a relatively coincidental pattern of potential distribution in their native areas (Fig. 5c, d \& e), which was similar to that of A. obscuriceps (Fig. 5b). The suitable LGM area of the host plant species So. sorbifolia showed slight differences compared with the other plants, indicating that suitable areas excluded Taiwan and expanded moderately toward the north in Japan (Fig. 5f). Notably, our LGM distribution model maps of A. obscuriceps and the four related host plants exhibited similar habitat gaps as geographical barriers on the exposed ECS continental shelf between China and Japan (Fig. 5b, c, d, e \& f). Outside of the native range areas, highly suitable areas of $A$. obscuriceps identified by ENM included the northeastern areas along the Pacific coast, the eastern states in North America, Western Europe, areas around the Black Sea, South Eastern Australia, most of New Zealand, Central and Southern Africa, and New Guinea (Additional file 11: Figure S7). Areas in Southern Brazil and Chile and those along the Pacific coast of Columbia, Ecuador and Peru also showed high climate suitability (Additional file 11: Figure S7). Our niche model successfully identified the currently disjunct distribution pattern of $A$. obscuriceps on both sides of 

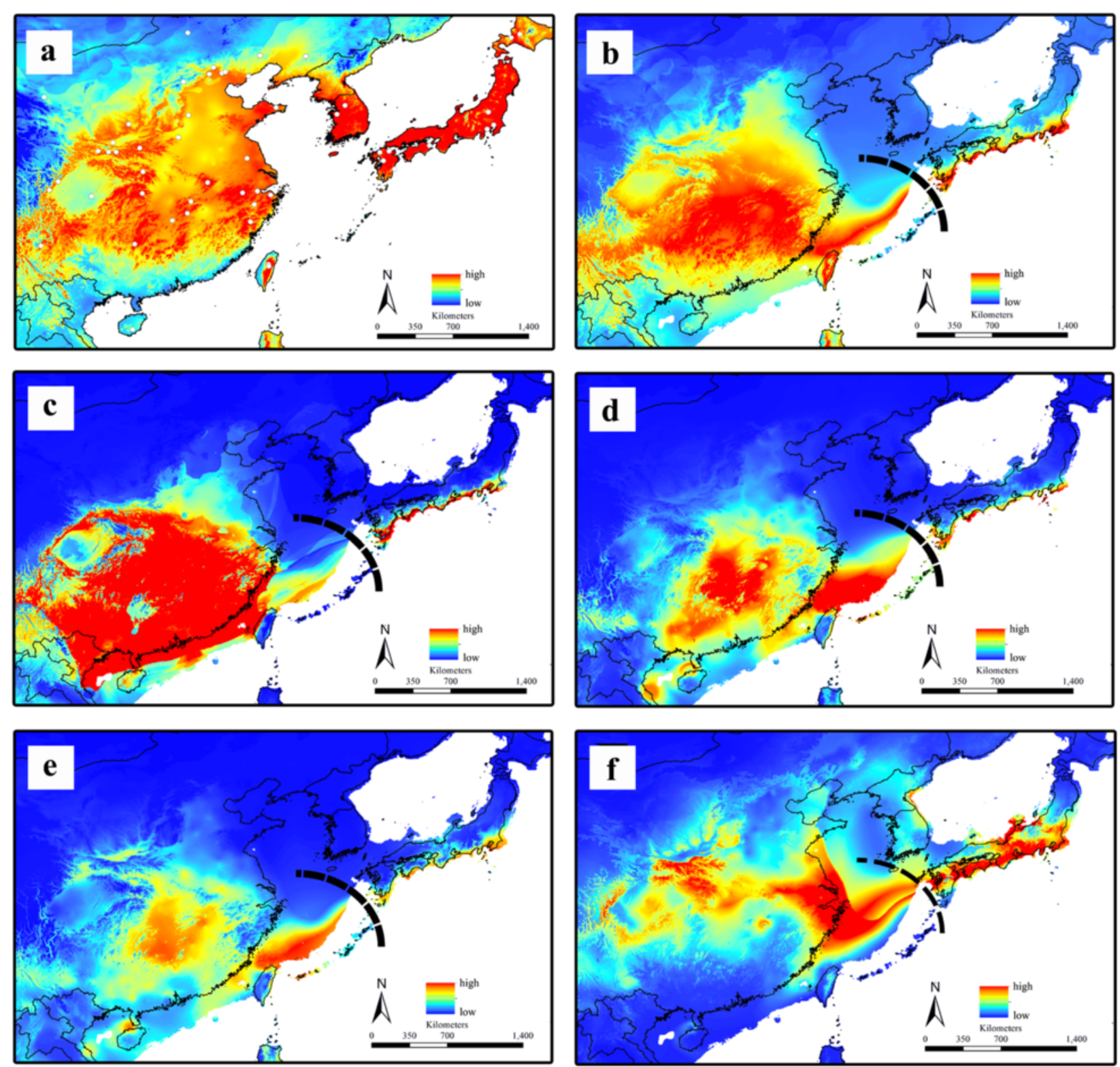

Fig. 5 Modelled suitable areas of A. obscuriceps and related four host plants across ECS. a The current suitable areas of A. obscuriceps. b The LGM suitable areas of $A$. obscuriceps. c The LGM suitable areas of Castanea mollissima. $\mathbf{d}$ The LGM suitable areas of Salix babylonica. e The LGM suitable areas of Salix matsudana. f The LGM suitable areas of Sorbaria sorbifolia. LGM, the Last Glacial Maximum (21,000 - 18,000 year BP). Black bold lines represent habitat gap/barrier

the Atlantic Ocean through comparisons with the actual invasion records, which indicated the predictive power of our model. In addition, the actual records of the four host plants probably matched the areas of potential invasion of A. obscuriceps outside the native areas (Additional file 12: Figure S8).

\section{Discussion}

Divergence time and underlying factors

The results of mitochondrial and nuclear DNA showed that $A$. obscuriceps comprised two main phylogenetic lineages, one from China and one from Japan, with reciprocally exclusive haplotypes that occupied distinct geographic distributions in China and Japan. The major genetic and geographical isolations achieved in our study were similar to several other species including the temperate plant Kalopanax septemlobus (Thunb.) Koidz., 1784 [48], the horseshoe bat Rhinolophus ferrumequinum (Schreber, 1774) [49], and the cotton pest Adelphocoris suturalis (Jakovlev, 1882) [50]. Based on the results of phylogenetic and haplotype network analyses, both sides of the ECS shared private haplotypes but did not share 
ancestral haplotypes, which suggested that divergence could have alternatively occurred long time ago and no substantial gene flow between the two regions were detectable since the divergence time, in accordance with the result of IMa2. Therefore, dispersal from China to Japan (or vice versa) through the hypothesized dispersal corridor (e.g., ECS land bridge) during the Pleistocene could have rarely occurred. However, the molecular results were inclined to disclose the more realistic situation that a former ancestor was extensively distributed in the pan-China-Japan region and was divided into two isolated groups because of the submergence of the ECS land bridge, as generally expected under a mode of vicariance/ allopatric differentiation [51]. From late Miocene to Pleistocene, three periods of ECS land bridge formation and connection occurred between the Eurasian continent and the Japanese Islands at approximately 5.0-7.0 Ma, 1.3-2.0 Ma and 0.015-0.2 Ma respectively [52]. During these periods, most of the wide continental shelves of the ECS were exposed. Although the divergence time between the Chinese and Japanese lineages estimated by the IMa2 (i.e., 1.07 [0.79-1.32] Ma) varied in a relatively extensive range because of uncertainty in the mutation rate of mitochondrial DNA, our estimated divergence time and its HPD intervals approximately fell into the period of the "Ryukyu Coral Sea Stage" (0.2-1.3 Ma). During this period, the ECS sea level increased rapidly, and the dominantly exposed ECS land bridge area became entirely submerged [53]. Based on these paleo-data, we propose that the vicariance scenario led to divergent lineages of $A$. obscuriceps on both sides of the ECS. The ancestral status of $A$. obscuriceps was probably extensively distributed in East Asia, including the ECS land bridge in the early Pleistocene. At approximately $0.79-1.32 \mathrm{Ma}$, lineage differentiation between the population in China and that in Japan was driven by population contraction and extinction on the ECS land bridge because of sea level increase and land bridge submergence. However, we could not verify whether the ancestral status of $A$. obscuriceps was extensively distributed across East Asia in the early Pleistocene in this scenario. This phenomenon indicated that the cold and arid climates during the Plio-Miocene to early Pleistocene were less extensive than that during the middle and late Pleistocene [54]. Indeed, our LGM distribution model for $A$. obscuriceps showed that the potential range of the species contracted moderately in the Chinese region and sharply shrank toward the south coastal zone in the Japanese region. This finding suggested that $A$. obscuriceps was relatively sensitive to the low temperature in the northern regions during the LGM. Therefore, the Plio-Miocene to early Pleistocene environment might have created more favorable conditions for the ancestral status of $A$. obscuriceps to occupy relatively extensive and continuous regions in East Asia.
The results of phylogenetic and haplotype network analyses showed that several individuals from Taiwan were nested structures within the haplogroups of China, which indicated that a relatively shallow divergence occurred between the populations in Mainland China and that in Taiwan. The shared ancestral haplotypes showed that recent gene flow also existed between these two regions. The divergence time (i.e., $0.15[0.10-0.22]$ $\mathrm{Ma}$ ) between Mainland China and Taiwan approximated the penultimate glaciation period $(0.13-0.30 \mathrm{Ma})$ [55], which was located at the transition time from the midPleistocene to the late Pleistocene. The earth, particularly the Northern Hemisphere, experienced a sudden climate shift during the period called the "mid-Pleistocene revolution" [56]. The mid-Pleistocene climate revolution was characterized by a gradual change in the dominant climate periodicity from $40 \mathrm{ka}$ to $100 \mathrm{ka}$ centered on the marine isotope stage $23 / 22$ boundary at approximately 0.9 million years [57]. This sudden shift in climate cycles resulted in increasing durations of cold periods, followed by fluctuation of the East Asian monsoon, which had profound effects on vegetation and creature responses [58]. Therefore, we speculate that the cold environment during the transition from the midPleistocene to late Pleistocene induced the differentiation of A. obscuriceps populations between Mainland China and Taiwan.

\section{Inferences of the historical demography of $A$. obscuriceps in the native range}

Following the inferred sundering in the early-middle Pleistocene, populations of $A$. obscuriceps experienced different demographic histories in Mainland China, Japan, and Taiwan. For the regions in Mainland China and Japan, the observed pairwise differences of mtDNA haplotypes fit the sudden expansion model particularly well (Fig. 4a \& b), producing significantly negative neutrality test statistics (Table 3). This finding provides strong evidence of a recent demographic expansion in these two regions, which is also in line with the results of EBSP (Additional file 8: Figure S4a, b). Population expansion occurred approximately 0.14 Ma years ago and subsequently sustained a steady growth with the increase in population size over time, which coincided with the Last Interglacial (LIG) (0.12-0.14 Ma BP). A direct link between range expansion and the beginning of the warm climate seems plausible. Temperatures during the LIG were approximately $2-5{ }^{\circ} \mathrm{C}$ warmer than those in the present ECS region [48]. Thus, the warm and moist East Asian summer monsoons intensified [59], allowing the persistence of vegetation similar to that observed today [60], which consequently might also lead to an increase in suitable habitats for $A$. obscuriceps throughout the warmer periods of the LIG. During the LGM period, the 
ice sheets did not cover most of East Asia and the glacial cycle effects were less dramatic [61]. Thus, the mild climate relative to Europe and North America might have also mitigated the demographic stresses on $A$. obscuriceps. Maintenance of the large effect of population size during cooler periods might be also partially attributed to the population genetic structure. Theoretically, although several habitats were lost and fragmented because of the cold glacial period, the census size of populations may still be able to highly inflate as a result of frequent migration events among subpopulations due to strong dispersal ability [62]. Our results of the reticular haplotype network and nonsignificant IBD parameters indicate that this species possesses relatively strong dispersal ability for frequent gene flow, which conforms to the theoretical description. In this study, we proposed that, if $A$. obscuriceps was sheltered in multiple refugia during the LGM, then frequent migration among populations at all geographic distances might have occurred through its strong dispersal ability. Such continuous gene flow could have made A. obscuriceps less sensitive to habitat reduction and fragmentation driven by climate changes.

In contrast to Mainland China and Japan, the population in Taiwan has a relatively small size, which is consistent with the result of EBSP (Additional file 8: Figure S4c). The multimodal mismatch distributions, together with the nonsignificant parameter statistics (Fig. 4c; Table 3), indicate a long-term persistence of the Taiwan population in separate refugia without significant growth [29]. The only significant migration detected by the IMa2 was from Mainland China to Taiwan. This recent gene flow might have mitigated the divergence between the Mainland China and Taiwan populations, which also corresponded to the ancestral haplotype networks (Fig. 2). The lack of local ancestral haplotypes and the large number of private haplotypes, together with permanently small population sizes, indicate that $A$. obscuriceps in Taiwan might have experienced more severe extinctions and/or stronger genetic drift.

\section{Host plant habitat requirement as the key to $A$. obscuriceps colonization}

Many factors influence the establishment of a nonindigenous species in a community, including physiological flexibility (e.g., dispersal ability and habitat requirement) [63]. The ENM can identify areas of matching climate space occupied by the invasive species in the native area. Prior to inferring potential areas, the ENM seeks to identify suitable climate spaces for species, without considering dispersal ability [40]. The Mantel tests did not reveal significant IBD patterns for $A$. obscuriceps (Fig. 3), indicating a strong dispersal ability, high gene flow at all geographic distances, and minimal genetic drift. The results of haplotype networks and AMOVA (Fig. 2; Table 2) also indicate complex and higher levels of reticulation within Chinese and Japanese lineages, which are characterized by higher gene flow among populations within a group at all geographic distances. Based on these results, the small and winged insect A. obscuriceps was likely to be a strong flier with presumably strong capacity for long-distance dispersal. The ENM prediction results showed that suitable habitats of this species were distributed in the partial continental shelves of the ECS and the south coastal zone of Japan, but with a short disjunct distance between them (Fig. 5b). Depending on its strong dispersal ability, A. obscuriceps could be expected to generate gene flow between these two regions. However, this standpoint was rejected because of the significantly divergent lineages between the populations in China and Japan (Fig. 2). Instances of this type of situation were observed in the steppe bird species Tetrax tetrax (Linnaeus, 1758) [64] and two grasshopper species Hesperotettix viridis (Thomas, 1872) and Ceracris kiangsu Tsai, 1929 [63, 65]. This puzzling issue might be sorted out by focusing on the habitat requirement of this species. The highly suitable areas during the LGM predicted by the ENM of the four host plants showed a similar distribution pattern, which was consistent with the predicted area of A. obscuriceps: similar unsuitable area gaps existed as the geographical barrier between the ECS continental shelf and Japanese regions (Fig. $5 \mathrm{c}$, d, e \& f). Thus, we speculated that the discontinuous distribution of the four host plants shaped a habitat filter that probably impeded the gene flow of $A$. obscuriceps between the populations in China and Japan. From another point of view, this hypothesis could be supported by evidence from populations in Mainland China and Taiwan. Individuals of Taiwan were partially nested structures within haplogroups of China (Fig. 2), indicating a recent limited gene flow that probably occurred after the divergence event. The LGM prediction showed that the suitable habitat of $A$. obscuriceps occupying a continuous range covered the southeast coastal areas of Mainland China and Taiwan across the Taiwan Strait, which shaped a large and intact refuge. Coincidentally, the same distribution patterns were detected in the LGM prediction of the three host plants C. mollissima, Sa. babylonica and Sa. matsudana (Fig. 5c, d \& e), indicating continuous suitable areas across the Taiwan Strait. Thus, the continuous distribution of these host plants possibly shaped a habitat corridor, which was coupled with a moderate dispersal ability and promoted gene flow between the populations in Mainland China and Taiwan.

\section{Conclusions}

Phylogeography coupled with ENM revealed the Pleistocene history of the invasive species A. obscuriceps in its native range. Molecular data revealed a significant genetic 
differentiation between the populations in China and Japan. Divergence time approximately coincided with the period of sea level increase, and recent gene flow was not detected by the IMa2 analysis. We determined that the vicariance scenario led to divergent lineages of A. obscuriceps on both sides of the ECS. No significant IBD relationship was observed between $\Phi s t$ and the Euclidean distances in the Mantel tests, which is consistent with the hypothesis that this species has a good dispersal ability. Our LGM niche modeling of the four related host plants exhibited similar habitat gaps on the exposed ECS continental shelf between China and Japan, but showed continuous distribution across the Taiwan Strait. We provided a successful example of uncovering the genetic structure and underlying factors of an invasive insect across the ECS. The findings of our study will also shed light on the potential role of habitat requirement in the process of biological invasion.

\section{Additional files}

Additional file 1: Table S1. PCR primer sequences used to amplify mitochondrial and nuclear fragments. (DOC $33 \mathrm{~kb}$ )

Additional file 2: Table S2. Pearson's correlation coefficient $(R)$ for the 7 bioclimatic variables. (DOC $36 \mathrm{~kb}$ )

Additional file 3: Table S3. Discovery of Amphiareus obscuriceps in different countries and related reference. (DOC $44 \mathrm{~kb}$ )

Additional file 4: Figure S1. Phylogenetic Bayesian tree analysis of all 182 haloptypes from mitochondrial markers (COI + COIl + CytB). Branches above $50 \%$ Bayesian posterior probabilities are shown in number. (DOC $53 \mathrm{~kb}$ )

Additional file 5: Figure S2. Phylogenetic ML tree analysis of all 182 haloptypes from mitochondrial markers (COI + COII + CytB). ML bootstrap percentages above $50 \%$ bootstrap support are shown in number. (DOC $41 \mathrm{~kb})$

Additional file 6: Figure S3. Zones of genetic discontinuities. Barriers were retained under the majority-rule criteria according to their importance. (a \& b) Mitochondrial data. (c \& d) Nuclear data. (DOC $1636 \mathrm{~kb}$ )

Additional file 7: Table S4. Nucleotide polymorphisms in each geographic population. S, number of segregating sites; NHap, number of haplotypes; $H d$, haplotype diversity; $\pi$, nucleotide diversity. (DOC $60 \mathrm{~kb}$ )

Additional file 8: Figure S4. Extended Bayesian Skyline Plots (EBSP), inferred from mitochondrial and nuclear DNA sequence variations (COI, CytB, ITS1) depicting changes in effective population size (Ne) as a function of time for Mainland China (a), Japan (b), Taiwan (c), and the whole set (d). The thick solid black line marks the estimated medians, and the area delimited by the upper and lower grey lines represents the HPD 95 \% confidence intervals for Ne. (DOC $117 \mathrm{~kb}$ )

Additional file 9: Figure S5. Divergence time among the three mitochondrial haplogroups (Mainland China, Japan and Taiwan) calculated by BEAST. The area delimited by blue lines represents the HPD $95 \%$ confidence intervals. (DOC $58 \mathrm{~kb}$ )

Additional file 10: Figure S6. Jackknife of regularized training gain for A. obscuriceps. (DOC $36 \mathrm{~kb}$ )

Additional file 11: Figure S7. Niche model based on native records of A. obscuriceps and those transferred worldwide using Maxent. Dark red color represents higher suitability, while dark blue indicates lower suitability. Black and red dots represent the native and invasive records respectively. (DOC $111 \mathrm{~kb}$ )
Additional file 12: Figure S8. Coordinates of four host plants' positions on the map of predicted invasive areas of $A$. obscuriceps. Dark red color represents higher suitability, while dark blue indicates lower suitability. Black and red dots represent the native and invasive records respectively. (a) Castanea mollissima; (b) Salix babylonica; (c) Salix matsudana; (d) Sorbaria sorbifolia. (DOC $1211 \mathrm{~kb}$ )

\section{Acknowledgements}

The authors wish to thank Professor Jintang Dong, Qiang Xie and Dávid Rédei of Nankai University for reviewing the manuscript and providing helpful comments. The third author $\mathrm{KY}$ is much indebted to the following individuals for providing Japanese materials: Dr. Tadashi Ishikawa of Tokyo University of Agriculture, Japan; Mr. Tatsuya Nozaki of Fukuoka, Japan; Mr. Teruaki Ban of Hokkaido, Japan; and Mr. Noriyuki Muro of University of Tokyo, Japan.

\section{Funding}

Funding for this research was provided by the National Basic Research Program of China (2013CB127600), the National Natural Science Foundation of China (Nos. 31430079, J1210005, and 31501853), and the Postdoctoral Science Foundation of China (2015 M570225).

Availability of data and materials

The datasets supporting the results of this article are available in the Dryad repository, doi:10.5061/dryad.pr4d0.

\section{Authors' contributions}

DZ carried out the molecular genetic studies. ZY contributed to ecological niche modeling analysis. ZY, KY, YZ, and CZ participated in data analyses and helped prepare the additional files. DZ and ZY conducted molecular data analyses and drafted the manuscript. WB directed this study and revised the manuscript. All authors read and approved the final manuscript.

\section{Competing interests}

The authors declare that they have no competing interests.

\section{Consent for publication}

Not applicable.

Ethics approval and consent to participate

No specific permits were required for the insect specimens collected for this study in China and Japan. Insect specimens were collected with a net from deciduous trees and shrub plants. The field studies did not involve endangered or protected species. Flower bugs Amphiareus obscuriceps are common small insects, and are not included in the "List of Protected Animals in China and Japan".

\section{Author details}

Institute of Entomology, College of Life Sciences, Nankai University, 94 Weijin Road, Tianjin 300071, China. ${ }^{2}$ Tokushima Prefectural Museum, Bunka-no-Mori Park, Hachiman-chô, Tokushima 770-8070, Japan.

Received: 1 March 2016 Accepted: 18 August 2016

Published online: 31 August 2016

\section{References}

1. Holt BG, Lessard JP, Borregaard MK, Fritz SA, Araújo MB, Dimitrov D, et al. An update of Wallace's zoogeographic regions of the World. Science. 2013:339:74-8.

2. Voris HK. Maps of Pleistocene sea levels in Southeast Asia: shorelines, river systems and time durations. J Biogeogr. 2000;27:1153-67.

3. Lambeck K, Esat TM, Potter EK. Links between climate and sea levels for the past three million years. Nature. 2002;419:199-206.

4. Siddall M, Rohling EJ, Almogi-Labin A, Hemleben C, Meischner D, Schmelzer I, et al. Sea-level fluctuations during the last glacial cycle. Nature. 2003;423:853-8.

5. Chen D, Zhang X, Kang H, Sun X, Yin S, Du H, et al. Phylogeography of Quercus variabilis Based on Chloroplast DNA Sequence in East Asia: Multiple Glacial Refugia and Mainland-Migrated Island Populations. PLoS One. 2012;7(10):e47268 
6. Guo X, Zhao D, Jung $D$, Li Q, Kong LF, Ni G, et al. Phylogeography of the Rock Shell Thais clavigera (Mollusca): Evidence for Long-Distance Dispersal in the Northwestern Pacific. PLoS One. 2015;10(8):e0135540.

7. Ye Z, Zhu GP, Chen PP, Zhang DL, Bu WJ. Molecular data and ecological niche modelling reveal the Pleistocene history of a semi-aquatic bug (Microvelia douglasi douglasi) in East Asia. Mol Ecol. 2014;23:3080-96.

8. Qi XS, Yuan N, Comes HP, Sakaguchi S, Qiu YX. A strong 'filter' effect of the East China Sea land bridge for East Asia's temperate plant species: inferences from molecular phylogeography and ecological niche modelling of Platycrater arguta (Hydrangeaceae). BMC Evol Biol. 2014;14:1.

9. Ye JW, Guo XD, Wang SH, Bai WN, Bao L, Wang HF, et al. Molecular evidence reveals a closer relationship between Japanese and mainland subtropical specimens of a widespread tree species, Acer mono. Biochem Syst Ecol. 2015;60:143-9.

10. Phillipsen IC, Kirk EH, Bogan MT, Mims MC, Olden JD, Lytle DA. Dispersal ability and habitat requirements determine landscape-level genetic patterns in desert aquatic insects. Mol Ecol. 2015;24:54-69.

11. Derycke S, Remerie T, Backeljau T, Vierstraete A, Vanfleteren J, Vincx M, et al. Phylogeography of the Rhabditis (Pellioditis) marina species complex: evidence for long-distance dispersal, and for range expansions and restricted gene flow in the northeast Atlantic. Mol Ecol. 2008;17:3306-22.

12. Rocha LA, Bass AL, Robertson DR, Bowen BW. Adult habitat preferences, larval dispersal, and the comparative phylogeography of three Atlantic surgeonfishes (Teleostei: Acanthuridae). Mol Ecol. 2002;11:243-52.

13. Podnar M, Bruvo Mađarić B, Mayer W. Non-concordant phylogeographical patterns of three widely codistributed endemic Western Balkans lacertid lizards (Reptilia, Lacertidae) shaped by specific habitat requirements and different responses to Pleistocene climatic oscillations. J Zool Syst Evol Res 2013:52:119-29.

14. Bu WJ, Zheng LY. Hemiptera. Lashiochilidae, Lyctocoridae, Anthocoridae. Fauna Sinica Insecta. Beijing: Science; 2001. p. 90-2.

15. Rabitsch W. Alien true bugs of Europe (Insecta: Hemiptera: Heteroptera). Zootaxa. 1827;2008:1-44.

16. Wu ZY, Peter HR, Hong DYE. Flora of China (English edition). Beijing: Science; 1994

17. Friess T. Libellen (Odonata) und Wanzen (Heteroptera) aus dem Naturschutzgebiet "Gut Walterskirchen" am Wörthersee. Carinthia II. 2000;190./110.:517-30.

18. Henry TJ, Wheeler Jr AG, Steiner Jr WE. First North American records of Amphiareus obscuriceps (Poppius) (Hemiptera: Heteroptera: Anthocoridae), with a discussion of dead-leaf microhabitats. Proc Entomol Soc Wash. 2008;110:402-16.

19. Larkin MA, Blackshields G, Brown NP, Chenna R, McGettigan PA, McWilliam H, et al. ClustalW and ClustalX version 2.0. Bioinformatics. 2007;23:2947-8.

20. Hall TA. BioEdit: a user-friendly biological sequence alignment editor and analysis program for Windows 95/98/NT. Nucleic Acids Symp Ser. 1999;41:95-8.

21. Ronquist $F$, Teslenko $M$, van Der Mark $P$, Ayres $D$, Darling $A$, Höhna $S$, et al. MrBayes 3.2: efficient Bayesian phylogenetic inference and model choice across a large model space. Syst Biol. 2011;61:539-42.

22. Silvestro D, Michalak I. raxmIGUI: a graphical front-end for RAxML. Org Divers Evol. 2012;12:335-7.

23. Lanfear R, Calcott B, Ho SY, Guindon S. Partitionfinder: combined selection of partitioning schemes and substitution models for phylogenetic analyses. Mol Biol Evol. 2012;29:1695-701.

24. Rozas J, Sánchez-DelBarrio JC, Messeguer X, Rozas R. DnaSP, DNA polymorphism analyses by the coalescent and other methods. Bioinformatics. 2003;19:2496-7.

25. Bandelt HJ, Forster P, Röhl A. Median-joining networks for inferring intraspecific phylogenies. Mol Biol Evol. 1999;16:37-48.

26. Excoffier $L$, Lischer HEL. Arlequin suite ver 3.5: a new series of programs to perform population genetics analyses under Linux and Windows. Mol Eco Resour. 2010;10:564-7.

27. Jensen JL, Bohonak AJ, Kelley ST. Isolation by distance, web service. BMC Genet. 2005;6:13

28. Manni F, Guérard E, Heyer E. Geographic patterns of (genetic, morphologic, linguistic) variation: how barriers can be detected by "Monmonier's algorithm". Hum Biol. 2004;76:173-90.

29. Rogers AR, Harpending H. Population growth makes waves in the distribution of pairwise genetic differences. Mol Biol Evol. 1992;9:552-69.

30. Drummond AJ, Rambaut A. BEAST: Bayesian evolutionary analysis by sampling trees. BMC Evol Biol. 2009;7:214.
31. Ho SYW, Shapiro B. Skyline-plot methods for estimating demographic history from nucleotide sequences. Mol Ecol Resour. 2011;11:423-34.

32. Andersen NM, Cheng L, Damgaard J, Sperling FAH. Mitochondrial DNA sequence variation and phylogeography of oceanic insects (Hemiptera: Gerridae: Halobates spp.). Mar Biol. 2000;136:421-30.

33. Damgaard J, Zettel H. Genetic diversity, species phylogeny and historical biogeography of the Aquarius paludum group (Heteroptera: Gerridae). Insect Syst Evol. 2003;34:313-27.

34. Pfeiler E, Bitler BG, Ramsey JM, Palacios-Cardiel C, Markow TA. Genetic variation, population structure, and phylogenetic relationships of Triatoma rubida and T. recurva (Hemiptera: Reduviidae: Triatominae) from the Sonoran Desert, insect vectors of the Chagas' disease parasite Trypanosoma cruzi. Mol Phylogenet Evol. 2006;41:209-21.

35. Bargues MD, Marcilla A, Ramsey JM. Nuclear rDNA-based Molecular Clock of the Evolution of Triatominae (Hemiptera: Reduviidae), Vectors of Chagas Disease. Mem Inst Oswaldo Cruz. 2000;95:567-73.

36. Posada D, Crandall KA. Modeltest: testing the model of DNA substitution. Bioinformatics. 1998;14:817-8.

37. Rambaut A, Suchard MA, Xie D, Drummond AJ. Tracer v1.6, 2014. Available from http://beast.bio.ed.ac.uk/Tracer.

38. Hey J. Isolation with migration models for more than two populations. Mol Biol Evol. 2010;27:905-20.

39. Hey J, Nielsen R. Multilocus methods for estimating population sizes, migration rates and divergence time, with applications to the divergence of Drosophila pseudoobscura and D. persimilis. Genetics. 2004;167:747-60.

40. Li L, Yu GY, Mcavoy TJ, Reardon RC, Wu Y, Salom SM, et al. The biological characters, habitat and food seletion of Tetraphleps galchanoides (Hemiptera: Anthocoridae). Acta Entomol Sin. 2011;54:800-8.

41. Zhu G, Bu W, Gao Y, Liu G. Potential Geographic Distribution of Brown Marmorated Stink Bug Invasion (Halyomorpha halys). PLoS One. 2012;7(2):e31246.

42. Hijmans RJ, Cameron SE, Parra JL, Jones PG, Jarvis A. Very high resolution interpolated climate surfaces for global land areas. Int I Climatol. 2005:25:1965-78.

43. Phillips SJ, Anderson RP, Schapire RE. Maximum entropy modeling of species geographic distributions. Ecol Model. 2006;190:231-59.

44. Collins WD, Bitz CM, Blackmon ML, Bonan GB, Bretherton CS, Carton JA, et al. The Community Climate System Model version 3 (CCSM3). J Clim. 2006;19:2122-43.

45. Fawcett T. An introduction to ROC analysis. Pattern Recogn Lett. 2006;27:861-74.

46. Slatkin M, Hudson RR. Pairwise comparisons of mitochondrial-DNA sequences in stable and exponentially growing populations. Genetics. 1991;129:555-62.

47. Harpending HC. Signature of ancient population growth in a low-resolution mitochondrial-DNA mismatch distribution. Hum Biol. 1994;66:591-600.

48. Sakaguchi S, Qiu YX, Liu YH. Climate oscillation during the Quaternary associated with landscape heterogeneity promoted allopatric lineage divergence of a temperate tree Kalopanax septemlobus (Araliaceae) in East Asia. Mol Ecol. 2012:21:3823-38.

49. Flanders J, Wei L, Rossiter SJ, Zhang SY. Identifying the effects of the Pleistocene on the greater horseshoe bat, Rhinolophus ferrumequinum, in East Asia using ecological niche modelling and phylogenetic analyses. J Biogeogr. 2011;38:439-52.

50. Zhang L, Li H, Li S, Zhang A, Kou F, Xun H, et al. Phylogeographic structure of cotton pest Adelphocoris suturalis (Hemiptera: Miridae): strong subdivision in China inferred from mtDNA and rDNA ITS markers. Sci Rep. 2015;5:14009.

51. Kropf M, Comes HP, Kadereit JW. Long-distance dispersal vs vicariance: the origin and genetic diversity of alpine plants in the Spanish Sierra Nevada. New Phytol. 2006;172:169-84.

52. Kimura M. Land connections between Eurasian continent and Japanese Islands - related to human migration. Migrat and Diffus. 2003:4:14-33.

53. Kimura M. Quaternary paleogeography of the Ryukyu Arc. J Geogr 1996;105:259-85.

54. Hao QZ, Guo ZT, Qiao YS, Xu B, Oldfield F. Geochemical evidence for the provenance of middle Pleistocene loess deposits in southern China. Quaternary Sci Rev. 2010;29:23-4.

55. Zhao J, Shi Y, Wang J. Comparison between quaternary glaciations in china and the marine oxygen isotope stage (MIS): an improved schema. Acta Geogr Sin. 2011;66:867-84.

56. Adams J, Maslin M, Thomas T. Sudden climate transitions during the Quaternary. Prog Phys Geog. 1999;23:1-36. 
57. Berger W, Bickert T, Jansen E, Wefer G, Yasuda M. The central mystery of the Quaternary Ice Age. Oceanus. 1993;36:53-6.

58. Hayashi R, Takahara H, Tanida K. Vegetation response to East Asian monsoon fluctuations from the penultimate to last glacial period based on a terrestrial pollen record from the inland Kamiyoshi Basin, western Japan. Palaeogeogr Palaeocl. 2009;284:246-56.

59. Han WX, Fang XM, Berger A. Tibet forcing of mid-Pleistocene synchronous enhancement of East Asian winter and summer monsoons revealed by Chinese loess record. Quaternary Res. 2003;78:174-84.

60. Kelly MJ, Edwards RL, Cheng H, Yuan DX, Cai YJ, Zhang ML, et al. High resolution characterization of the Asian Monsoon between 146000 and 99 000 years BP from Dongge Cave, China and global correlation of events surrounding Termination II. Palaeogeogr Palaeocl. 2006;236:20-38.

61. Rost KT. Paleoclimatic field studies in and along the Qinling Shan (Central China). GeoJournal. 1994;34:107-20.

62. De A, Durrett R. Stepping-stone spatial structure causes slow decay of linkage disequilibrium and shifts the site frequency spectrum. Genetics. 2007;176:969-81.

63. Apple IL, Grace T, Joern A, Amand PS, Wisely SM. Comparative genome scan detects host-related divergent selection in the grasshopper Hesperotettix viridis. Mol Ecol. 2010;19:4012-28.

64. Garcia JT, Manosa S, Morales MB, Ponjoan A, De la Morena EG, Bota G, et al. Genetic consequences of interglacial isolation in a steppe bird. Mol Phylogenet Evol. 2011;61:617-76.

65. Fan Z, Jiang GF, Liu YX, Blanchard B, He QX. Population Explosion in the Yellow-Spined Bamboo Locust Ceracris kiangsu and Inferences for the Impact of Human Activity. PLoS One. 2015;10(2):e0118110.

\section{Submit your next manuscript to BioMed Central and we will help you at every step:}

- We accept pre-submission inquiries

- Our selector tool helps you to find the most relevant journal

- We provide round the clock customer support

- Convenient online submission

- Thorough peer review

- Inclusion in PubMed and all major indexing services

- Maximum visibility for your research

Submit your manuscript at www.biomedcentral.com/submit
Biomed Central 